\title{
Metal Nanoparticles Based Electrochemical Biosensors for Cholesterol
}

\author{
Sameena Mehtab*, MGH Zaidi, Pragati Joshi
}

Department of Chemistry, College of Basic Sciences and Humanities, G.B. Pant University of Agriculture and Technology, Pantnagar, U.S Nagar-263145, Uttarakhand, India

\begin{abstract}
Electrochemical biosensors (EBS) have recently gained much attention in the field of health care for the management of cholesterol $(\mathrm{CHO})$. This review comprises a report on the modification of electrode surfaces with metal nanoparticles (MNP) for selective and sensitive sensing of CHO. Quantitative analysis of cholesterol is carried out with the use of the amperometric data of linear calibration plot. A good synergistic between MNP of working electrode (WE) and CHO give fast electron transfer and précised electrochemical detection of CHL.
\end{abstract}

\section{GRAPHICAL ABSTRACT}
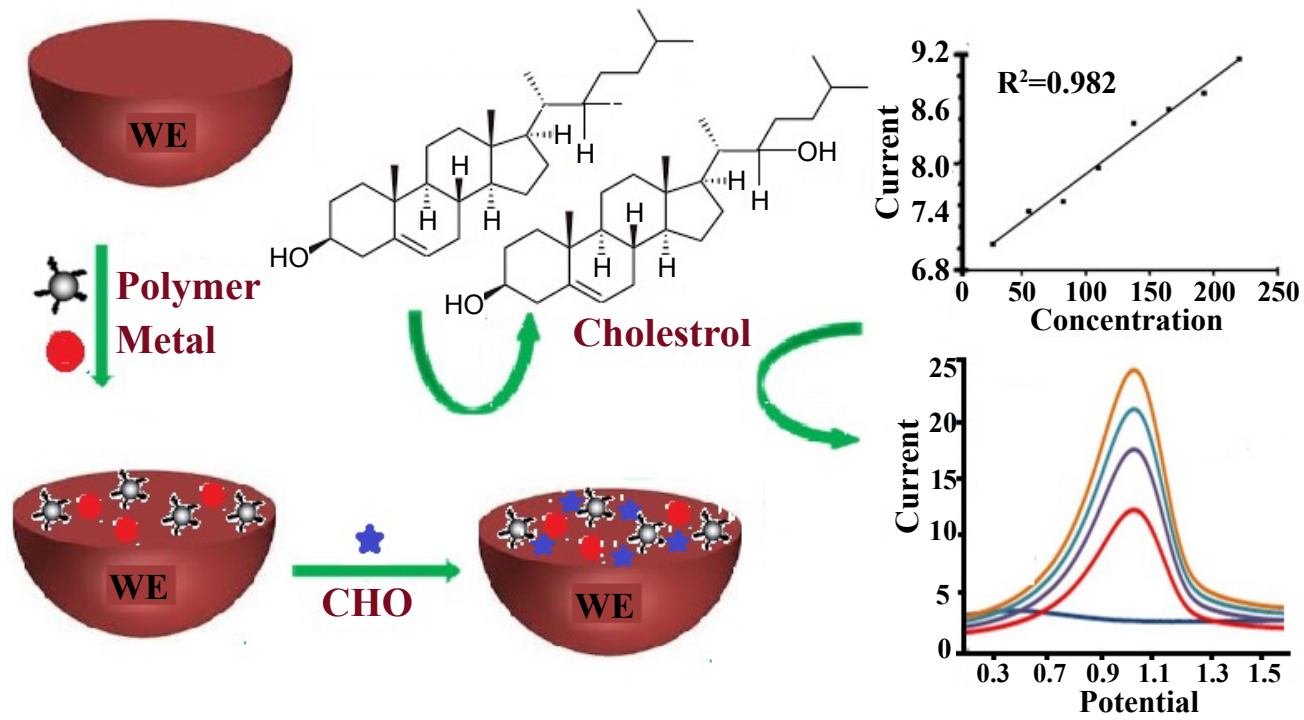

Keywords: Biosensor; Cholesterol; Electrochemical; Metal nanoparticles; Working electrode

\section{INTRODUCTION}

Monitoring of $\mathrm{CHO}$ in blood is very important to control health issues related to its abnormal levels. Electrochemical methods has received immense acceptance selectivity for CHL sensing, because of reasonable simplicity, low cost, a wide working range and high sensitivity. EBS is an analytical device integrating the specificity of biomolecules with electronics based physico-chemical transducer for conversion of a biochemical signal into quantifiable electrical signal. A conventional EBS mainly contains three electrodes a saturated $\mathrm{Ag} / \mathrm{AgCl}$ electrode and a platinum wire served as reference and counter electrodes, respectively and glassy carbon electrode or metal plate electrode that serve as WE. It is required to focus on the chemical modifications of WE surface to improve its selective sensing for a target molecule. In the fabrication of EBS, cholesterol oxidase $(\mathrm{ChOx})$ is most commonly used as the biosensing element that remain immobilized with different type of electroactive materials. Due to high conducting properties of MNP with different binders, they have been effectively immobilized with $\mathrm{ChOx}$ and used for surface modification of WE to sense $\mathrm{CHO}$. To improve the EBS performance, MNP have been reported in various combinations with viz. conductive polymers, graphene, chitosan and graphene oxide carbon nanotubes etc., which endow high electrical conductivity, effective surface area, and fast

${ }^{*}$ Correspondence to: Sameena Mehtab, Department of Chemistry, College of Basic Sciences and Humanities, G.B. Pant University of Agriculture and Technology, Pantnagar, U.S Nagar-263145, Uttarakhand, India, Tel: +91-7055670603; E-mail: smiitr@gmail.com

Received: October 24, 2019, Accepted: January 03, 2020, Published: January 10, 2020

Citation: Mehtab S, Zaidi MGH, Joshi P (2020) Metal Nanoparticles Based Electrochemical Biosensors for Cholesterol. J Nanomed Nanotech. 11:540. doi: $10.35248 / 2157-7439.19 .11 .540$

Copyright: () 2020 Mehtab S, et al. This is an open-access article distributed under the terms of the Creative Commons Attribution License, which permits unrestricted use, distribution, and reproduction in any medium, provided the original author and source are credited. 
electron-transfer rate. The EBS efficiency mainly depends on the modification of working electrode surface with different electro active coating materials. The morphology of the prepared WE were characterized by scanning electron microscopy, transmission electron microscopy, atomic force microscopy, X-ray diffraction and energy dispersive X-ray spectroscopy. The physicochemical properties of the modified electrode at each stage of the construction were characterized by electrochemical impedance spectroscopy and cyclic voltammetry. The performance of electrodes towards detection and quantification of CHL are investigated through square wave voltammetry and differential pulse voltammetry methods. In the present review we compiled various types of MNP based EBS for trace level $\mathrm{CHO}$ estimation.

\section{MNP BASED CHOLESTEROL BIOSENSORS}

MNP have good electronic properties and thus explored in the fabrication of various biosensors [1]. Pt/Graphene and $\mathrm{Pt} /$ CNT decorated electrodes were developed for amperometric measurement was used to detect $\mathrm{CHO}$ upto $0.5 \mathrm{nM}[2,3]$. The specific molecular recognition of $\mathrm{CHO}$ upto nanolevel by digitonin gold nanoparticles was reported [4]. Electrochemical-enzymatic sensor by selective enzyme immobilization on nano-sized carbon interdigitated electrodes decorated with gold nanoparticles claim CHO estimation with LOD $1.28 \mu \mathrm{M}$ [5]. Pt-Bi combined with the organic enzyme platform, demonstrates effective $\mu \mathrm{M}$ biosensing of $\mathrm{CHO}$ [6]. Silver nanowires, graphene oxide film electrodes were formed and data reveals high sensitivity [7]. Excellent sensitivity of $\mathrm{CHO}$ was achieved from biosensor based on an enzyme-immobilized microtubular $\mathrm{ZnO} / \mathrm{ZnS}$ heterostructure [8]. Electrochemical photoelectrochemical $\mathrm{CHO}$ biosensor based on graphene embedded titanium nanowires was also reported with remarkable sensitivity of $6 \mu \mathrm{M}$ [9]. $\mathrm{CHO}$ biosensors based on graphene oxide and Pd nanostructure was successfully used for the analysis of total cholesterol in human serum and butter [10]. Nanocomposite of molybdenum disulfide nanoparticles were employed for CHO ultra-quantification [11].

\section{CONCLUSION}

The current advances in electrochemical biosensors is characterized by an application-directed approach and leads to miniaturized biosensors by transforming working electrode surface. This article reviews electrochemical $\mathrm{CHO}$ biosensors and different methods proposed for their construction to overcome their limitations in selectivity and sensitivity.

\section{REFERENCES}

1. Shrivastava S, Jadon N, Jain R. Next-generation polymer nanocomposite-based electrochemical sensors and biosensors: A review. TrAC Trends in Analytical Chemistry. 2016;82:55-67.

2. Dey RS, Raj CR. Development of an amperometric cholesterol biosensor based on graphene-Pt nanoparticle hybrid material. The Journal of Physical Chemistry C. 2010;114(49):21427-21433.

3. Yang J, Lee H, Cho M, Nam J, Lee Y. Nonenzymatic cholesterol sensor based on spontaneous deposition of platinum nanoparticles on layer-by-layer assembled CNT thin film. Sensors and Actuators B: Chemical. 2012;171:374-337.

4. Raj V, Jaime R, Astruc D, Sreenivasan K. Detection of cholesterol by digitonin conjugated gold nanoparticles. Biosensors and Bioelectronics. 2011;27(1):197-200.

5. Sharma D, Lee J, Seo J, Shin H. Development of a sensitive electrochemical enzymatic reaction-based cholesterol biosensor using nano-sized carbon interdigitated electrodes decorated with gold nanoparticles. Sensors. 2017;17(9):2128.

6. Soorya VC, Sheela B. Flower like Bi structures on Pt surface facilitating effective cholesterol Biosensing. Materials Science and Engineering: C. 2016;64:183-189.

7. Xu L, Hou Y, Zhang M, Cheng $\mathrm{T}$, Huang W, Yao C, et al. Electrochemical sensor based on a silver nanowires modified electrode for the determination of cholesterol. Analytical Methods. 2015;7(13):5649-5653.

8. Giri AK, Charan C, Saha A, Shahi, VK, Panda, AB. An amperometric cholesterol biosensor with excellent sensitivity and limit of detection based on an enzyme-immobilized microtubular ZnO@ZnS heterostructure. Journal of Materials Chemistry A. 2014;2:16997. 17004 .

9. Komathi S, Muthuchamy N, Lee KP, Gopalan AI. Fabrication of a novel dual mode cholesterol biosensor using titanium dioxide nanowire bridged 3D graphene nanostacks. Biosensors and Bioelectronics. 2016;84:64-71.

10. Dey RS, Raj CR. Enzyme-integrated cholesterol biosensing scaffold based on in situ synthesized reduced graphene oxide and dendritic Pd nanostructure. Biosensors and Bioelectronics. 2014;62:357-364.

11. Wang TY, Du KZ, Liu WL, Zhang JX, Li MX. Electrochemical sensors based on molybdenum disulfide nanomaterials, Electroanalysis. 2015;27:2091-2097. 